\title{
Epidemiological characteristics and outcomes of COVID-19 in diabetic versus non-diabetic patients
}

\author{
Leila Moftakhar $^{1} \cdot$ Parisa Moftakhar $^{2} \cdot$ Elahe Piraee $^{3} \cdot$ Haleh Ghaem $^{4} \cdot$ Aliasghar Valipour $^{5} \cdot$ Habibollah Azarbakhsh $^{6}$
}

Received: 23 November 2020 / Accepted: 20 January 2021 / Published online: 9 February 2021

(C) Research Society for Study of Diabetes in India 2021

\begin{abstract}
Objectives and background People with diabetes (PWD) are one of the high-risk groups for coronavirus disease 2019 (COVID19) infection, increasing the disease mortality. This study was aimed to compare the epidemiological characteristics and outcomes of COVID-19 in diabetic versus non-diabetic individuals.

Methods In this retrospective observational study, the epidemiological characteristics of the two groups of diabetic $(n=1365)$ and non-diabetic $(n=15,026)$ subjects with definite diagnosis of COVID-19 in the southwestern region of Iran were compared. All clinical signs and comorbidities of the patients were evaluated. Chi-square test was used to examine the differences in qualitative variables between diabetic and non-diabetic groups.

Results Of 16,391 enrolled subjects, $8.3 \%$ had diabetes, and 28.3\% of COVID-19-related deaths occurred in diabetics. Also, the mortality rate among diabetics was reported as $14.3 \%$. The average age of diabetic patients and non-diabetic patients was 59 and 37 years, respectively. The odds of fever, cough, shortness of breath, headache, and underlying diseases, such as hypertension, cardiovascular disease, chronic lung disease, immune deficiency, and hyperlipidemia, were significantly higher in diabetic patients than in non-diabetics.

Conclusion Diabetes is associated with increased mortality rate in patients with COVID-19 and is considered as a major risk factor for COVID-19 infection, posing a major public health challenge for health policymakers in managing and controlling the disease. Therefore, development of prevention and treatment strategies aimed at reducing COVID-19 morbidity and mortality in diabetes patients is of significant importance.
\end{abstract}

Keywords COVID- $19 \cdot$ Diabetes $\cdot$ Mortality

\section{Introduction}

Diabetes is the fifth leading cause of death throughout the world (approximately 3 million deaths per year), which is mainly caused by defects in either insulin secretion or action. The prevalence of diabetes has dramatically increased over the past two decades and is projected to increase from 285 million in 2010 to 438 million in 2030 [1]. Also, according to the
Habibollah Azarbakhsh

azarbakhshhabibollah@gmail.com

Leila Moftakhar

moftakhar_p@yahoo.com

Parisa Moftakhar

moftakhar.parisa@yahoo.com

Elahe Piraee

nazgolelahe@gmail.com

Haleh Ghaem

ghaemh@sums.ac.ir

Aliasghar Valipour

aliasgharvalipour698@gmail.com
Abadan Faculty of Medical Sciences, Abadan, Iran

2 Department of Clinical Sciences, Faculty of Veterinary Medicine, Shahid Chamran University of Ahvaz, Ahvaz, Iran

3 Student Research Committee, Shiraz University of Medical Sciences, Shiraz, Iran

4 Research Center for Health Sciences, Department of Epidemiology, School of Health, Shiraz University of Medical Sciences, Shiraz, Iran

5 Department of Public Health, Abadan Faculty of Medical Sciences, Abadan, Iran

6 Student Research Committee, Shiraz University of Medical Sciences, Shiraz, Iran 
World Health Organization, the prevalence of diabetes in Iran will increase from 3 million in 2010 to 6 million in 2030 [2]. People with diabetes (PWD) are one of the high-risk groups for Coronavirus Disease 2019 (COVID-19) due to their weakened immune system [3] .

According to the World Health Organization, COVID-19 highlighted on December 30, 2019 in Wuhan, China, and quickly infected many Chinese people [4]. On January 30, 2020, WHO identified corona as a global public health concern [5]. The family of coronaviruses is the cause of a variety of well-known diseases occurring in humans, ranging from common cold to Middle Respiratory Syndrome (MERS) and Acute Severe Respiratory Syndrome (SARS), and currently COVID-19 as a new problematic member of this family $[6,7]$. Symptoms of the virus range from mild to severe, including fever, cough, and difficulty breathing [8]. PWD are at higher risk of infection, especially flu and pneumonia, and with proper blood sugar control, the risk can be reduced among these individuals. In fact, it was observed that diabetes was a leading cause of death in patients with the 2009 pandemic influenza (H1N1), SARS, coronavirus, and MERS [9]. In a study conducted in Wuhan on 140 patients with COVID-19, diabetes was not a risk factor for severe COVID-19 [10]. However, another study on 150 patients (68 deaths and 82 recovered patients) in Wuhan showed diabetes as an important predictor of COVID-19 [11]. Analysis of 11 studies on patients with COVID-19 showed that hyperglycemia and diabetes were not predictors of severe COVID-19 [12]. However, among 72,314 reported cases of COVID-19 by the China Centers for Disease Control and Prevention, mortality in PWD showed an increasing trend (2.3\% and $7.3 \%$ of PWD in general) [13].

Due to the fact that limited information about the complications of COVID-19 in PWD is unknown, the present study was carried out to answer the question whether COVID-19 is more severe in PWD with higher mortality rate. This study was aimed to investigate and compare the epidemiological characteristics and outcomes of COVID-19 in patients with diabetes mellitus and non-diabetic patients.

\section{Methods}

This retrospective observational study was performed on 16,391 patients with COVID-19 who were under care in the southwestern region of Iran (Khuzestan province) from March to September 2020. The total population of this region was estimated 627,970 based on the annual growth, using the databases of province health centers and the national census of 2020. Confirmation of definite COVID-19 cases by RT-PCR was performed using nasal and throat swab samples. Patients with normal vital signs (pulse, respiration rate, and blood pressure) with saturated oxygen levels above $93 \%$ and mild symptoms were sent to home quarantine and monitored daily by health professionals. Patients were hospitalized with severe symptoms such as chest pain, shortness of breath, and a saturated oxygen level of less than $90 \%$. Patients with saturated oxygen levels between 90 and $93 \%$ were admitted to hospital or undergoing home quarantine, depending on the physicians' opinion and other clinical conditions. In the study area, there were only two hospitals that, like other medical centers in the country, performed all stages of testing and hospitalization of patients according to the protocols and national guidelines of corona management. Also, according to the patients' clinical condition, according to the doctor's prescription, a CT scan was performed for some patients to diagnose the extent of lung involvement.

All clinical and demographic information of patients, as well as the results of daily follow-up and the final outcome of each patient, were recorded by health experts. Duplicate samples have been identified and removed based on the national code of participants. Subjects were divided into two groups: diabetic group $(n=1365)$ and non-diabetic group $(15,026)$. First, demographic characteristics, symptoms, and other comorbid diseases were compared between diabetic and non-diabetic groups; then, the role of comorbid diseases in mortality and survival of all study subjects was compared. Finally, diabetic and non-diabetic patients were compared in terms of clinical symptoms and comorbid diseases. Variables used in this study include age, gender, final outcome (e.g., death and survival), symptoms (e.g., negative stenosis, fever, cough, lack of smell, lack of taste, fatigue, muscle pain, diarrhea, sore throat, and headache), and comorbidities, such as hypertension, diabetes, cardiovascular disease, kidney disease, liver disease, immune deficiency, high blood fats, and chronic lung disease.

\section{Statistical analyses}

Descriptive quantitative data were expressed as mean and median, and qualitative data were expressed as percentage and frequency. The distribution of continuous variables was performed using Kolmogorov-Smirnov test. Mann-Whitney test was also used to analyze abnormal data analysis. Chi-square test and Fisher's exact test were used to evaluate the differences between qualitative variables in the mortality of two study groups. Data analysis was performed using SPSS version 19.0. The significance level was set as 0.05 .

\section{Results}

During the study period, a total of 16,391 cases of COVID-19 occurred in the cities affiliated to Abadan University of Medical Sciences. The average age of the patients was 38 years, with an interquartile range of 30-51 years. A total of $54.55 \%$ of the patients were male, and $62.60 \%(n=10,256)$ 
had a history of contact with definite or suspected cases of COVID-19. Also, $4.21 \%$ of the patients $(n=691)$ died, of whom $28.36 \%(n=196)$ had diabetes (Table 1$)$. The most common symptoms displayed by subjects were fever $(68.70 \%)$, cough $(67.80 \%)$, and shortness of breath $(49.30 \%)$. The most common comorbidities in all patients were hypertension (5.50\%), cardiovascular disease (5\%), chronic lung disease $(1.90 \%)$, and chronic liver disease $(1.70 \%)$ (Table 1$)$.

Of all participants, 1365 (8.32\%) were diabetic and 15,026 (91.68\%) were non-diabetic. Also, $55.50 \%$ of diabetic patients were female, and $755(55.30 \%)$ had a history of contact with definite or suspected cases of COVID-19. Compared with non-diabetic patients, diabetic patients were older, with an average age of 59 years (IQR: 49-67) vs. 37 years (IQR 2949), and risk of comorbidities was higher in diabetic patients than in non-diabetic individuals, including hypertension as $418(30.60 \%)$ vs. $478(3.20 \%)$, heart disease as 266 $(19.50 \%)$ vs. 549 (3.70\%), chronic kidney disease as 72 $(5.30 \%)$ vs. $213(1.40 \%)$, chronic lung disease as 56
(4.10\%) vs. $258(1.70 \%)$, chronic liver disease as $13(1 \%)$ vs. $27(0.20 \%)$, immunodeficiency as $24(1.80 \%)$ vs. 63 $(0.40 \%)$, and hyperlipidemia as $18(1.30 \%)$ vs. $34(0.20 \%)$.

Fever, cough, and shortness of breath were significantly higher in diabetic patients compared with non-diabetic patients, and sore throat, chest pain, and reduced sense of taste and smell were significantly higher in non-diabetic patients than in diabetic patients. Diabetic patients had higher mortality ( $14.40 \%$ in diabetic patients vs. $3.30 \%$ in non-diabetic). The history of contact with definite or suspected cases of COVID-19 was higher in non-diabetic subjects (63.20\% vs. $55.30 \%$ ) (Table 1).

In addition, 15,700 patients $(95.78 \%)$ survived from COVID-19. Compared with COVID-19 survivors, nonsurvivors were significantly older (median age of 66 years (IQR 55-76) versus 38 years (IQR 30-50)). Chronic diseases were significantly higher in non-survivors, including diabetes as $196(28.40 \%)$ patients vs. $1169(7.44 \%)$, hypertension as $143(20.70 \%)$ vs. $753(4.79 \%)$, heart disease as $142(20.50 \%)$
Table 1 The characteristics of patients with COVID-19 with or without diabetes

\begin{tabular}{|c|c|c|c|c|}
\hline Variable & $\begin{array}{l}\text { Total }(n=16,391) \\
\text { Number }(\%)\end{array}$ & $\begin{array}{l}\text { Diabetes }(n=1365) \\
\text { Number }(\%)\end{array}$ & $\begin{array}{l}\text { Non-diabetes }(n=15,026) \\
\text { Number }(\%)\end{array}$ & $p$ value \\
\hline Age, median (IQR) & $38(30-51)$ & $59(49-67)$ & $37(29-49)$ & $<0.001$ \\
\hline \multicolumn{5}{|l|}{ Gender } \\
\hline Male & $8940(54.55)$ & $607(44.50)$ & $8333(55.50)$ & \multirow[t]{2}{*}{$<0.001$} \\
\hline Female & $7446(45.45)$ & $758(55.50)$ & $6688(44.50)$ & \\
\hline \multicolumn{5}{|l|}{ Symptoms } \\
\hline Fever & $11258(68.70)$ & $1075(78.80)$ & $10183(67.80)$ & $<0.001$ \\
\hline Cough & $11106(67.80)$ & $1035(75.80)$ & $10071(67.00)$ & $<0.001$ \\
\hline Dyspnea & $8086(49.30)$ & $910(66.70)$ & $7176(47.80)$ & $<0.001$ \\
\hline Muscular pain & $1520(9.30)$ & $124(9.10)$ & $1396(9.30)$ & 0.801 \\
\hline Diarrhea & $351(2.10)$ & $26(1.90)$ & $325(2.20)$ & 0.528 \\
\hline Nausea & $436(2.70)$ & $39(2.90)$ & $397(2.60)$ & 0.636 \\
\hline Sore throat & $1239(7.60)$ & $79(5.80)$ & $1160(7.70)$ & 0.010 \\
\hline Anorexia & $78(0.50)$ & $6(0.40)$ & $72(0.50)$ & 0.839 \\
\hline Headache & $618(3.80)$ & $57(4.20)$ & $561(3.70)$ & 0.270 \\
\hline Fatigue & $54(0.30)$ & $3(0.20)$ & $51(0.30)$ & 0.460 \\
\hline Chest pain & $121(0.70)$ & $4(0.30)$ & $117(0.80)$ & 0.045 \\
\hline Decreased sense of smell & $378(2.30)$ & $10(0.70)$ & $368(2.40)$ & $<0.001$ \\
\hline Decreased sense of taste & $85(0.50)$ & $1(0.10)$ & $84(0.60)$ & 0.017 \\
\hline \multicolumn{5}{|l|}{ Comorbidities } \\
\hline Hypertension & $896(5.50)$ & $418(30.60)$ & $478(3.20)$ & $<0.001$ \\
\hline Cardiovascular disease & $815(5.00)$ & $266(19.50)$ & $549(3.70)$ & $<0.001$ \\
\hline Immunodeficiency & $87(0.50)$ & $24(1.80)$ & $63(0.40)$ & $<0.001$ \\
\hline Chronic kidney disease & $285(1.70)$ & $72(5.30)$ & $213(1.40)$ & $<0.001$ \\
\hline Chronic pulmonary disease & $314(1.90)$ & $56(4.10)$ & $258(1.70)$ & $<0.001$ \\
\hline Chronic liver disease & $40(0.20)$ & $13(1.00)$ & $27(0.20)$ & $<0.001$ \\
\hline Hyperlipidemia & $52(0.30)$ & $18(1.30)$ & $34(0.20)$ & $<0.001$ \\
\hline Exposure to disease & $10256(62.60)$ & $755(55.30)$ & $9501(63.20)$ & $<0.001$ \\
\hline Mortality & $691(4.20)$ & $196(14.40)$ & $495(3.30)$ & $<0.001$ \\
\hline
\end{tabular}


vs. $673(4.28 \%)$, immunodeficiency as 14 (2.20\%) vs. 72 $(0.45 \%)$, chronic kidney disease as $43(6.20 \%)$ vs. 242 $(1.54 \%)$, and chronic lung disease as 31 (4.50\%) vs. 283 $(1.80 \%)$ (Table 2).

Of 1365 diabetic patients with COVID-19, COVID-19 non-survivors were considerably older than survivors (median age of 66 years (IQR 57-73) vs. 57 years (IQR 48-66)). The risk of heart disease was higher in non-survivors (56 patients (28.57\%) vs. 193 patients $(16.50 \%))$. Gender, other comorbidities, and disease symptoms showed no significant difference with survived diabetic patients and non-survived patients (Table 3).

\section{Discussion}

This was a retrospective observational study, performed on 16,391 patients with COVID-19 in southwestern Iran. This study compared clinical features and mortality between people with type 2 diabetes and non-diabetic patients. Since the onset of COVID-19 pandemic, the disease has become widespread throughout the world, affecting a large number of people. The most vulnerable groups against COVID-19 are those with comorbid diseases, including diabetes. Various epidemiological evidences have shown that PWD are at higher risk for infectious diseases, such as measles and mumps [14]. Also, according to the results of previous studies, diabetes is one of the most common comorbidities associated with COVID-19. In our study, $8.3 \%$ of COVID-19 patients had type 2 diabetes. Other studies showed various percentages of COVID-19 patients with type 2 diabetes, as 20\% [15], 52\% [16], 24.9\% [17], $14.5 \%$ [18], and 21.8\% [19]. However, a number of studies have shown that the severity and complications of COVID-19 disease and the need for ICU stay were higher in diabetic patients than in non-diabetic individuals $[9,14,15$, 17, 20-22].

According to the results of our study, the median age of COVID-19 patients who had diabetes was higher than COVID-19 patients without diabetes (59 vs. 37), which was consistent with the results of many previous studies $[17,19$, 23 ]. Since diabetes is a chronic disease and chronic diseases are more common at older age, which explains higher average age of COVID-19 patients with diabetes.

The results of our study showed that symptoms of COVID19 , including fever, shortness of breath, and cough, were mostly reported in diabetic patients than in non-diabetic patients, while chest pain, sore throat, and decreased sense of smell and taste were more common in non-diabetic COVID19 patients. In a study conducted in China, fever, dry cough, and fatigue were the most common symptoms in diabetic COVID-19 patients [23], and another study showed cough and fever as the most common symptoms in these patients [24]. A systematic review study indicated that no significant differences in signs and symptoms of these differences may be due to variation in selected sample sizes in various studies, study population, and type of collected data [18]. As such, some studies have examined only patients with severe form of COVID-19, while others have examined patients with various severity of the disease. Undoubtedly, patients with different severities of the disease display different symptoms.

According to the results of our study, COVID-19-related mortality was higher in PWD than in non-diabetic individuals (14.4\% vs. $3.3 \%$ ). Also, a study performed on 46 cases of COVID-19 in Wuhan, China, showed that $42 \%$ of COVID19 deaths occurred among PWD. However, small sample size of this study should be considered in assessing the validity of the results. In another study on 72,312 people in China, mortality rate was higher in diabetic patients compared with non-
Table 2 The baseline characteristics of survivors and non-survivors infected with COVID-19

\begin{tabular}{lllll}
\hline Variable & $\begin{array}{l}\text { Total }(n=16,391) \\
\text { Number }(\%)\end{array}$ & $\begin{array}{l}\text { Survivors }(n=15,700) \\
\text { Number }(\%)\end{array}$ & $\begin{array}{l}\text { Non-survivors }(n=691) \\
\text { Number }(\%)\end{array}$ & $p$ value \\
\hline $\begin{array}{l}\text { Age, median (IQR) } \\
\text { Gender }\end{array}$ & $38(30-51)$ & $38(30-50)$ & $66(55-76)$ & $<0.001$ \\
Male & $8940(54.55)$ & $8424(54.70)$ & $378(54.70)$ & 0.986 \\
Female & $7446(45.45)$ & $6966(45.30)$ & $313(45.30)$ & \\
Comorbidities & & & $196(28.40)$ & $<0.001$ \\
Diabetes mellitus & $1365(8.30)$ & $1169(7.44)$ & $143(20.70)$ & $<0.001$ \\
Hypertension & $896(5.50)$ & $753(4.79)$ & $142(20.50)$ & $<0.001$ \\
Cardiovascular disease & $815(5.00)$ & $673(4.28)$ & $15(2.20)$ & $<0.001$ \\
Immunodeficiency & $87(0.50)$ & $72(0.45)$ & $43(6.20)$ & $<0.001$ \\
Chronic kidney disease & $285(1.70)$ & $242(1.54)$ & $31(4.50)$ & $<0.001$ \\
Chronic pulmonary disease & $314(1.90)$ & $283(1.80)$ & $2(0.30)$ & 0.797 \\
Chronic liver disease & $40(0.20)$ & $38(0.20)$ & $1(0.10)$ & 0.449 \\
Hyperlipidemia & $52(0.30)$ & $51(0.32)$ & & \\
\hline
\end{tabular}


Table 3 The baseline characteristics of survivors and non-survivors in patients with COVID-19 with diabetes

\begin{tabular}{|c|c|c|c|c|}
\hline Variable & $\begin{array}{l}\text { Total }(n=1365) \\
\text { Number }(\%)\end{array}$ & $\begin{array}{l}\text { Survivors }(n=1169) \\
\text { Number }(\%)\end{array}$ & $\begin{array}{l}\text { Non-survivors }(n=196) \\
\text { Number }(\%)\end{array}$ & $p$ value \\
\hline Age, median (IQR) & $59(49-67)$ & $57(48-66)$ & $66(57-73)$ & $<0.001$ \\
\hline \multicolumn{5}{|l|}{ Gender } \\
\hline Male & 607 (44.50) & $488(44.30)$ & $98(50.00)$ & \multirow[t]{2}{*}{0.141} \\
\hline Female & $758(55.50)$ & $613(55.70)$ & $98(50.00)$ & \\
\hline \multicolumn{5}{|l|}{ Symptoms } \\
\hline Fever & $1075(78.80)$ & $874(79.40)$ & $162(82.70)$ & 0.293 \\
\hline Cough & $1035(75.80)$ & $861(78.20)$ & $156(79.60)$ & 0.663 \\
\hline Dyspnea & $902(66.08)$ & $755(68.60)$ & $147(75.00)$ & 0.072 \\
\hline Muscular pain & $106(7.76)$ & $93(7.95)$ & $13(6.63)$ & 0.393 \\
\hline Diarrhea & $23(1.68)$ & $22(1.88)$ & $1(0.51)$ & 0.146 \\
\hline Nausea & $29(2.12)$ & $25(2.13)$ & $4(2.04)$ & 0.841 \\
\hline Sore throat & $68(4.98)$ & $63(5.38)$ & $5(2.55)$ & 0.066 \\
\hline \multicolumn{5}{|l|}{ Comorbidities } \\
\hline Hypertension & $391(28.64)$ & $324(27.71)$ & $67(34.18)$ & 0.181 \\
\hline Cardiovascular disease & $249(18.24)$ & $193(16.50)$ & $56(28.57)$ & $<0.001$ \\
\hline Immunodeficiency & $20(1.46)$ & $17(1.45)$ & $3(1.53)$ & 0.989 \\
\hline Chronic kidney disease & $71(5.20)$ & $57(4.87)$ & $14(7.14)$ & 0.265 \\
\hline Chronic pulmonary disease & $53(3.88)$ & $45(3.84)$ & $8(4.08)$ & 0.997 \\
\hline Chronic liver disease & $13(0.95)$ & $12(1.02)$ & $1(0.51)$ & 0.453 \\
\hline Exposure to disease & $690(50.54)$ & $599(51.24)$ & $91(46.42)$ & $0.039 \mathrm{D}$ \\
\hline
\end{tabular}

diabetic individuals [9]. The results of our study were supported by previous studies [17-19, 25] (17-40-42-12). The higher rate of mortality in diabetic patients can be due to their weakened immune system [26], poor lung function, and reduced lung capacity along with their common respiratory problems [18]. Furthermore, COVID-19 can affect diabetes pathogenicity as well as blood sugar control [20], all of which together can lead to increased mortality rate among these people. However, further research is needed to find out if COVID19-related mortality was actually caused by diabetes or other factors, as most of these people are elderly and may have at the same time some other chronic diseases, including hypertension and cardiovascular disease, which makes it a little difficult for us to identify the main cause of death due to COVID19. According to the findings of this study, the incidence of hypertension and heart disease was higher in PWD who died from COVID-19 than in non-diabetics. Other studies have shown that the mortality rate of COVID-19 was significantly higher in diabetic patients with heart disease as a comorbidity compared with non-diabetic patients.

\section{Conclusion}

The findings of this study suggest that diabetes increased mortality rate in patients with COVID-19 and is a major threat to these patients. Since COVID-19 is an unknown emerging disease, disease management has posed a huge challenge to health policymakers around the world. As a result, there is a need to develop prevention and treatment strategies for high-risk people, especially those with diabetes to reduce morbidity and mortality. It is also recommended that diabetic patients should take preventive measures, such as home quarantine, avoid unnecessary visits to medical centers, control blood glucose level, and follow the prevention guidelines developed by WHO experts for diabetics.

\section{Strengths and weaknesses}

Although, in our study, laboratory results and comprehensive information from patients were not available for further analysis, our study is one of the first studies in Iran, which examined clinical features of diabetic patients with COVID-19 and also, unlike many other similar studies, our study had a large sample size and also examined all cases of the disease, while many studies have been performed on only severe form of COVID-19.

We predict that we may have a diagnostic bias in our study. Because some people with asymptomatic or mild symptoms did not come to perform the test or the result of some tests was falsely negative, of course, our high sample size solves this problem 
Acknowledgments We would like to acknowledge the Department of Health of Abadan University of Medical Sciences.

Author contribution ML was responsible for the field working including data collection and management and wrote the discussion. PM was collected data and wrote the manuscript. $\mathrm{AbH}, \mathrm{PE}$, and $\mathrm{GHH}$ analysis data and wrote the manuscript. VA collected data and edited the final version of the manuscript.

Data Availability The data for the current study will not be shared publicly.

\section{Declarations}

\section{Consent for publication Not applicable.}

Competing interests The authors declare that they have no competing interests.

\section{References}

1. Moradi A, Hasani J. Comparative study of emotional regulation, self-control and defense mechanisms in cardiovascular patients, diabetic patients and normal people. Iranian J Psychiatr Nurs. 2019;6(1):43-52.

2. Shahbeik S, Taghavijoorabchi F, Abroshan F, Naseri M. Effectiveness of group-based cognitive therapy based on mindfulness on family function and marital adaptation of couples with type II diabetes. Iran J Nurs Res. 2019;13(6):68-75.

3. Singh AK, Singh A, Shaikh A, Singh R, Misra A. Chloroquine and hydroxychloroquine in the treatment of COVID-19 with or without diabetes: a systematic search and a narrative review with a special reference to India and other developing countries. Diabetes \& Metabolic Syndrome: Clinical Research \& Reviews. 2020.

4. Paraskevis D, Kostaki EG, Magiorkinis G, Panayiotakopoulos G, Sourvinos G, Tsiodras S. Full-genome evolutionary analysis of the novel corona virus (2019-nCoV) rejects the hypothesis of emergence as a result of a recent recombination event. Infection, Genetics and. Evolution. 2020;79:104212.

5. Amiri P. The role of electronic health during the Covid-19 crisis: a systematic review of literatures. J Health Biomed Inform. 2020;6(4):358-67.

6. Cherry JD, Krogstad P. Sars: The first pandemic of the 21 st century. Pediatr Res. 2004;56(1):1-5.

7. Pakzad B, Owlia MB. Corona Pandemic, Earth Restart Button. SSU J. 2020;28(1):2229-34.

8. Ezazi Bojnourdi E, Ghadampour S, Moradi Shakib A, Ghazbanzadeh R. Predicting corona anxiety based on cognitive emotion regulation strategies, health hardiness and death anxiety in diabetic patients. Iranian J Psychiatr Nurs. 2020;8(2):34-44.

9. Gupta R, Ghosh A, Singh AK, Misra A. Clinical considerations for patients with diabetes in times of COVID-19 epidemic. Diab Metab Syndr. 2020;14(3):211-2.

10. Zhang J-j, Dong X, Cao Y-y, Yuan Y-d, Yang Y-b, Yan Y-q, et al. Clinical characteristics of 140 patients infected with SARS-CoV-2 in Wuhan, China. Allergy. 2020.
11. Ruan Q, Yang K, Wang W, Jiang L, Song J. Clinical predictors of mortality due to COVID-19 based on an analysis of data of 150 patients from Wuhan, China. Intensive Care Med. 2020;46(5):8468.

12. Lippi G, Plebani M. Laboratory abnormalities in patients with COVID-2019 infection. Clin Chem Lab Med (CCLM). 2020;58(7):1131-4.

13. Wu Z, McGoogan JM. Characteristics of and important lessons from the coronavirus disease 2019 (COVID-19) outbreak in China: summary of a report of 72314 cases from the Chinese Center for Disease Control and Prevention. Jama. 2020;323(13): 1239-42.

14. Wang W, Lu J, Gu W, Zhang Y, Liu J, Ning G. Care for diabetes with COVID-19: advice from China. J Diabetes. 2020;12(5):417-9.

15. Huang C, Wang Y, Li X, Ren L, Zhao J, Hu Y, et al. Clinical features of patients infected with 2019 novel coronavirus in Wuhan, China. The lancet. 2020;395(10223):497-506.

16. Chen N, Zhou M, Dong X, Qu J, Gong F, Han Y, et al. Epidemiological and clinical characteristics of 99 cases of 2019 novel coronavirus pneumonia in Wuhan, China: a descriptive study. Lancet. 2020;395(10223):507-13.

17. Yan Y, Yang Y, Wang F, Ren H, Zhang S, Shi X, et al. Clinical characteristics and outcomes of patients with severe covid-19 with diabetes. BMJ Open Diabetes Res Care. 2020;8(1):e001343.

18. Abdi A, Jalilian M, Sarbarzeh PA, Vlaisavljevic Z. Diabetes and COVID-19: a systematic review on the current evidences. Diabetes Res Clin Pract. 2020;166:108347.

19. Kim MK, Jeon J-H, Kim S-W, Moon JS, Cho NH, Han E, et al. The clinical characteristics and outcomes of patients with moderate-tosevere coronavirus disease 2019 infection and diabetes in Daegu, South Korea. Diabetes Metab J. 2020;44(4):602-13.

20. Vas P, Hopkins D, Feher M, Rubino F. B Whyte M. Diabetes, obesity and COVID-19: a complex interplay. Diabetes Obes Metab. 2020;22(10):1892-6.

21. Liu Z, Li J, Huang J, Guo L, Gao R, Luo K, et al. Association between diabetes and COVID-19: a retrospective observational study with a large sample of 1,880 cases in Leishenshan Hospital, Wuhan. Front Endocrinol. 2020;11:478.

22. Guan W-j, Ni Z-y, Hu Y, Liang W-h, Ou C-q, He J-x, et al. Clinical characteristics of coronavirus disease 2019 in China. N Engl J Med 2020;382(18):1708-1720.

23. Wang F, Yang Y, Dong K, Yan Y, Zhang S, Ren H, et al. Clinical characteristics of 28 patients with diabetes and COVID-19 in Wuhan, China. Endocrine Practice. 2020

24. Li Y, Han X, Alwalid O, Cui Y, Cao Y, Liu J, et al. Baseline characteristics and risk factors for short-term outcomes in 132 COVID-19 patients with diabetes in Wuhan China: a retrospective study. Diabetes Res Clin Pract. 2020;166:108299.

25. Shang J, Wang Q, Zhang H, Wang X, Wan J, Yan Y, et al. The relationship between diabetes mellitus and COVID-19 prognosis: a retrospective cohort study in Wuhan, China. Am J Med. 2020.

26. Jeong I-K, Yoon KH, Lee MK. Diabetes and COVID-19: global and regional perspectives. Diabetes Res Clin Pract. 2020;166: 108303 .

Publisher's note Springer Nature remains neutral with regard to jurisdictional claims in published maps and institutional affiliations. 